\title{
Author Correction: Oxidative rearrangement of $(+)$-sesamin by CYP92B14 co-generates twin dietary lignans in sesame
}

\author{
Jun Murata (1) ', Eiichiro Ono², Seigo Yoroizuka ${ }^{3}$, Hiromi Toyonaga ${ }^{2}$, Akira Shiraishi ${ }^{1}$, Shoko Mori', \\ Masayuki Tera', Toshiaki Azuma', Atsushi J. Nagano ${ }^{4,5}$, Masaru Nakayasu6, Masaharu Mizutani ${ }^{6}$, \\ Tatsuya Wakasugi ${ }^{3}$, Masayuki P. Yamamoto ${ }^{3} \&$ Manabu Horikawa (i) ${ }^{1}$
}

Correction to: Nature Communications https://doi.org/10.1038/s41467-017-02053-7, published online 18 December 2017

The original version of the Supplementary Information associated with this article inadvertently omitted oligonucleotide primer sequences from Supplementary Table 3 and Supplementary Methods describing the molecular cloning of CYP92B14, CPR1 and CYP81Q cDNA fragments.

The HTML has been updated to include a corrected version of the Supplementary Information.

Published online: 25 May 2018

Open Access This article is licensed under a Creative Commons Attribution 4.0 International License, which permits use, sharing, adaptation, distribution and reproduction in any medium or format, as long as you give appropriate credit to the original author(s) and the source, provide a link to the Creative Commons license, and indicate if changes were made. The images or other third party material in this article are included in the article's Creative Commons license, unless indicated otherwise in a credit line to the material. If material is not included in the article's Creative Commons license and your intended use is not permitted by statutory regulation or exceeds the permitted use, you will need to obtain permission directly from the copyright holder. To view a copy of this license, visit http://creativecommons.org/licenses/by/4.0/.

(c) The Author(s) 2018

\footnotetext{
${ }^{1}$ Bioorganic Research Institute, Suntory Foundation for Life Sciences (SUNBOR), 8-1-1 Seikadai, Seika, Soraku, Kyoto 619-0284, Japan. ${ }^{2}$ Research Institute, Suntory Global Innovation Center Ltd (SIC), 8-1-1 Seikadai, Seika, Soraku, Kyoto 619-0284, Japan. ${ }^{3}$ Graduate School of Science and Engineering, University of Toyama, 3190 Gofuku, Toyama 930-8555, Japan. ${ }^{4}$ Faculty of Agriculture, Ryukoku University, 1-5 Yokotani, Seta Oe, Otsu, Shiga 520-2914, Japan. ${ }^{5}$ JST CREST, 4-1-8 Honcho, Kawaguchi, Saitama 332-0012, Japan. ${ }^{6}$ Graduate School of Agricultural Science, Kobe University, Kobe 657-8501, Japan. These authors contributed equally: Jun Murata, Eiichiro Ono, Seigo Yoroizuka. Correspondence and requests for materials should be addressed to M.P.Y. (email: mpyama@sci.u-toyama.ac.jp) or to M.H. (email: horikawa@sunbor.or.jp)
} 